\title{
The Design of Intelligent Lighting Monitoring System on ZigBee Based Wireless Personal Area Network
}

\author{
Dan Liu ${ }^{1,2}$ \\ ${ }^{1}$ College of Information Engineering \\ Dalian Ocean University \\ ${ }^{2}$ Key Laboratory of Marine Information Technology of \\ Liaoning Province \\ Dalian, China \\ e-mail: liudan@dlou.edu.cn \\ Xu Zhang \\ College of Network and Communication Engineering \\ Jinling Institute of Technology \\ Nanjing, China \\ e-mail:erik_zx@yahoo.cn
}

\author{
Fa-kui Ding \\ College of Information Engineering \\ Dalian Ocean University \\ Dalian, China \\ e-mail: m13342294122@gmail.com
}

\begin{abstract}
ZigBee is a technology now being deployed for wireless personal area networks. The paper discussed the technical characteristics and network topology structures of ZigBee. And then a design on intelligent lighting monitoring system based on ZigBee was proposed. The system adopted tree topology structure and used the chip CC2530 as the core, including hardware and software design. It could collect the light intensity signals of LEDs in real time; perform pulse width modulation and wireless communications between PAN coordinator, router and end devices. Supervisors could then assess the status of lighting by these parameters displaying on the personal computer. The article demonstrated the feasibility of the scheme through some corresponding experiments, where the LEDs could be controlled intelligently according to the indoor light intensity. Successful implementation provided a solution for convenient lighting work and thus saving energy, as well as cost. The results verified that ZigBee technology is an ideal solution to realize the wireless monitoring network.
\end{abstract}

Keywords-Lighting Monitoring; Wireless personal area network; ZigBee; CC2530; Router

\section{INTRODUCTION}

Recently, with rapid development of urban construction and enlarging lighting coverage, the traditional lighting management methods result in a series of environmental and energy problems such as wasted electrical energy because of the excess illumination, dispersing monitoring.ect. These cannot satisfy the requirements on power saving and efficiency improvement because of a great number of costs and poor real-time performance [12]. Hence, the intelligent monitoring and management of lighting is an important part and typical sector in the public lighting work. Using Zigbee techniques as a back bone to develop intelligent lighting system applications has been warming up in recent years. It essentially targets control and monitoring applications where relatively low levels of data throughput are needed, which is the best suited for designing intelligent lighting monitoring system.

A wireless personal area network (WPAN) is a human centered network, connecting personal communication devices in a spontaneous architecture, within a short-range, "personal" or "body" space. ZigBee is a technology now being deployed for wireless personal area networks. The mainly advantages of ZigBee technology lies in the following aspects: (a) reliable and self-configuration (b) large number of nodes supporting (c)easy to deploy (d)very long battery life(e) low cost.

The study applies the ZigBee wireless transmission module CC2530 as the core device to design an intelligent lighting monitoring system. The system can collect the light intensity signals of LEDs in real time and perform pulse width modulation (PWM).Supervisors can then assess the status of lighting by these parameters displaying on the personal computer. So the system realized the goals such as low cost, low consumption, and high automation, which changed the traditional management model, made the lighting monitoring management intelligent and efficient.

\section{IEEE802.15.4/ZIGBEE}

IEEE 802.15.4 standard [5] have been approved in 2003 as a physical (PHY) layer and multiple access control (MAC) layer for low data rate, low power, and low cost wireless personal area networks. ZigBee specification defines the network, security, and application framework profile layers for an IEEE 802.15.4-based system. The ZigBee Alliance [6-9] is an association of companies working together to enable reliable, efficient, wirelessly networked monitoring and controlling applications. The ZigBee is a higher-level protocol built on top of the IEEE802.15.4 physical and MAC layers of the protocol stack. 


\section{A. IEEE802.15.4}

At the PHY layer, IEEE 802.15.4 defines a total of 27 channels: 16 channels at a maximum rate of $250 \mathrm{kbps}$ in the SM 2.4-2.4835 GHz band, 10 channels at $40 \mathrm{kbps}$ in the SM $902-928 \mathrm{MHz}$ band, and 1 channel at $20 \mathrm{kbps}$ in the 68.0 - 868.6 MHz band. Carrier Sense Multiple Access with Collision Avoidance (CSMA/CA) mechanism is used at the MAC layer. IEEE 802.15.4 specified two device types: full function device (FFD) and reduced function device (RFD).

\section{B. ZigBee Specification}

The ZigBee network layer defines how the network formation is performed, how the network address is assigned to each node participating in the ZigBee network, routing discovery and routing maintain. ZigBee defines three types of devices in the network: ZigBee coordinator, ZigBee routers, and ZigBee end devices. An RFD can only be a ZigBee end device, while an FFD can be either a ZigBee coordinator or ZigBee router. The ZigBee coordinator is responsible for starting a new network. The routers have the ability of routing while the ZigBee end devices cannot participate in routing and have to rely on their corresponding ZigBee parent routers for routing which are either FFD or RFD.

\section{Topology structure}

Three network topologies are defined: star, tree and mesh topology in ZigBee network, as shown in Figure 1. In a star topology, the network is controlled by a single device (ZigBee coordinator) which corresponds to an IEEE 802.15.4 PAN coordinator. It is responsible for initiating and maintaining the devices on the network; all other devices, known as end-devices, directly communicate with the ZigBee coordinator. In mesh and tree topologies, the ZigBee coordinator is responsible for starting the network and for choosing certain key network parameters. The network may be extended through the use of ZigBee routers. In tree-topology networks, routers move data and control messages through the network by using a hierarchical routing strategy [10].

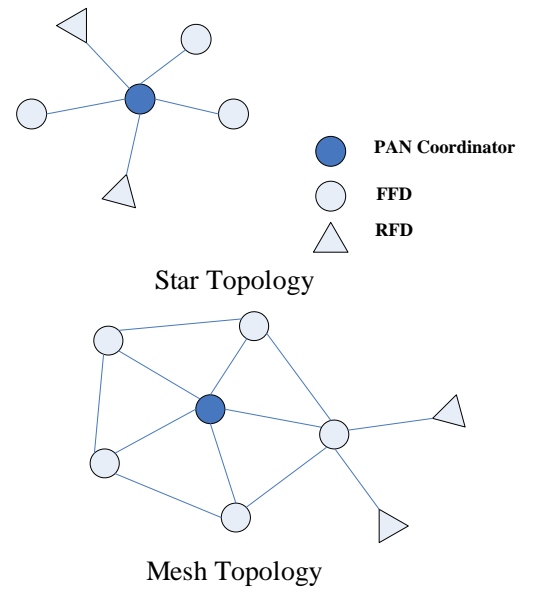

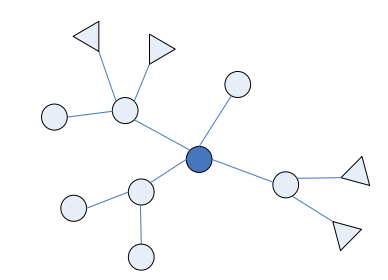

Tree Topology

Figure 1. Topology models

The tree topology of ZigBee is used in the design for lighting monitoring system in this paper.

\section{SOLUTION OF LIGHTING MONITORING SYSTEM}

As Figure 2. shows that this design adopted the tree topological network structure. The entire system is consisted of three parts which are named PAN coordinator, router and end devices. The PAN coordinator is connected with the monitor PC which is to form a ZigBee network to communicate with router. The photo sensors are designed on each end devices. The system can collect the light intensity signals of LEDs in real time; perform pulse width modulation and wireless communications between the PAN coordinator, router and end devices. Meanwhile it can display these lighting parameters on the personal computer.

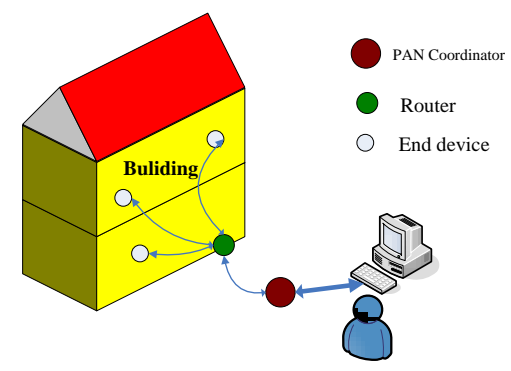

Figure 2. Architecture of the lighting monitoring system

\section{DESIGN FOR ZIGBEE-BASED LIGHTING MONITORING} SYSTEM

\section{A. Hardware design}

The hardware design for lighting monitoring system includes ZigBee CC2530 F149 module, thermal infrared sensors, photo sensors, LEDs, RS232 serial port communication cables, power and PC. The hardware structure is shown as figure 3 .

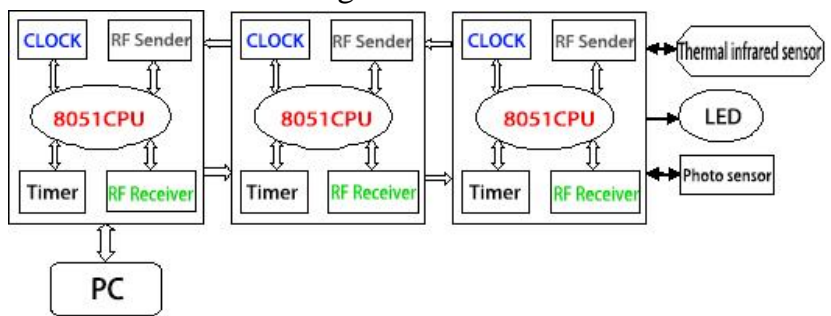

Figure 3. Hardware structure of the lighting monitoring system

The enhanced 8051MCU and RF module are integrated on the chip CC2530 that can provide a good solution for the smart control of lighting system based on ZigBee technology. The system consists of one sending part, some routers and some receiving modules. The sending part concludes a ZigBee PAN coordinator and a personal 
computer, while the router performs to forward data, and the receiving modules conclude some ZigBee end devices, sensors and LEDs. The system can collect the light intensity signals of LEDs in real time; perform pulse width modulation and logical and intelligent analysis. The signals are shown on the display and controlled according to these specific instructions. The main function of end devices is collecting environmental light intensity and transmitting to the router. The router forwards these data to the PAN coordinator, and then to PC. In the whole process of wireless communication, the PAN coordinator analyzes the data collected by the sensors and adjusts the duty cycle by the timers to perform LED lights control. The light of LEDs can be changed from brighter to darker or from darker to brighter according to indoor light intensity. Meanwhile the data could be displayed on monitor PC.

\section{B. Software design}

The indoor real-time light signals are collected by the photoconductors integrated on the ZigBee modules, which are forwarded by the router to the PAN coordinator, and then the PAN coordinator transmits them to the PC by the serial ports. Meanwhile the PC controls the LEDs according to the thresholds (190cd) in advance. The variable values of indoor light intensity are compared with the specific threshold. If the values are greater than $190 \mathrm{~cd}$, the LEDs will be turned off. If they are less than 190cd, meanwhile if anyone comes, the LEDs are on; if there is nobody, the LEDs are off. When the LEDs are on, they will be determined the brightness from off to very bright by PWM. In PWM, the light intensity values are divided into 5 levels to determine the brightness from off to very bright respectively: 210-190; 190-170; 170-150; 150-130; 130-110.

The corresponding key program of PWM is as follows: if(theMessageData[5]>90\&\&theMessageData[5]<=110) pwm=4;

if (theMessageData[5]> 70\&\&theMessageData $[5]<=90$ ) pwm=3;

if (theMessageData[5] $>50 \& \&$ theMessageData[5]<=70)

pwm=2;

if (theMessageData[5]>30\&\&theMessageData[5]<=50)

pwm $=1$;

if $($ theMessageData[5]>=10\&\&theMessageData[5]<=30) pwm $=0$;

void Timer_CallBack(uint8 timerId, uint8 channel, uint8 channelMode)

\{

if $($ counter $<6)$

counter++;

else

counter $=0$

if(counter<pwm)

HalLedSet

HAL_LED_3,

HAL_LED_MODE_ON ); else

$$
\text { HalLedSet ( }
$$

HAL_LED_3,
\}

The intelligent lighting monitoring system on ZigBee based wireless personal area network works as follows:

a) PAN Coordinator: The PAN coordinator initials the network by broadcasting a beacon frame with PAN identification. If there are nodes which are ready to join the network, it will assign the network addresses to the nodes and receive the data. If there is no node to join, the PAN coordinator will scan the beacon frame.

b) Router: After the initialization, the router sends a join request to the PAN coordinator in the network. The router will monitor network after joining the network successfully. If the there are some nodes to join the network, it will send the confirm information. If the router receives the lighting data rather than the join request, it will forward the data. Figure 4. shows the working flow.

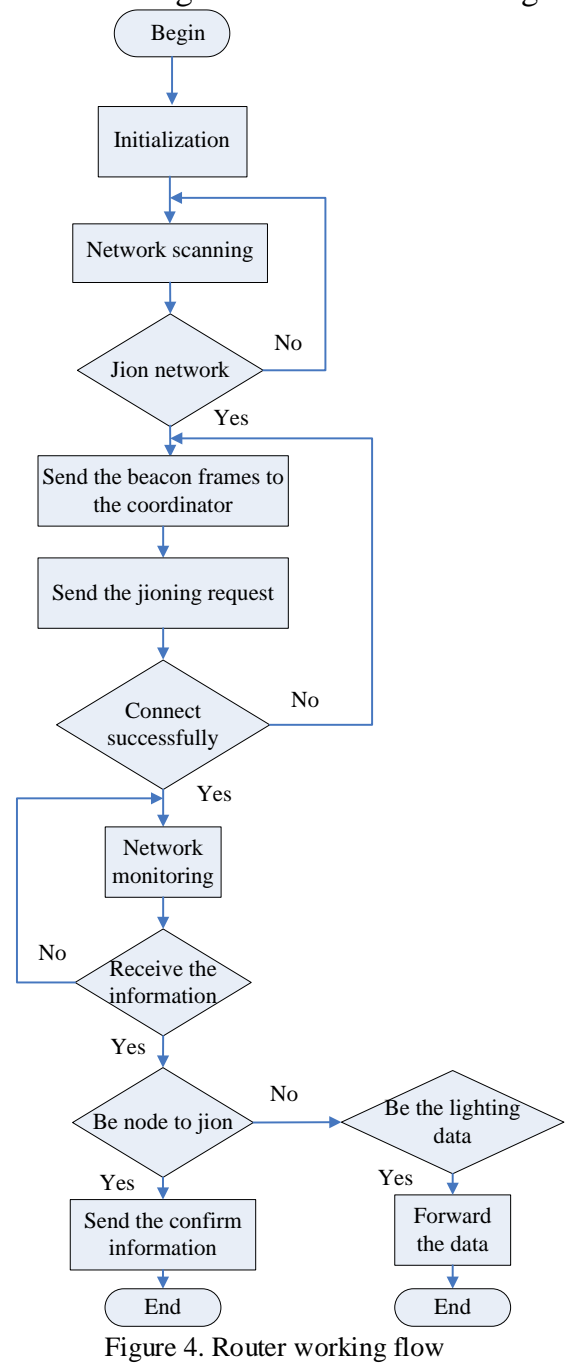

The corresponding key program of router is as follows:

PRIVATE bool_t IsMyDescendant uint16 parentaddr,uint 16 childaddr,uint8 parentdepth )

NWK_NeighborTable_s *ChildPtr;

if $($ dstaddr $==0 x f f f f)$

$\{*$ dstnexthop $=0 x$ ffff;

return TRUE;

\} 
\#ifdef I_AM_ENDDEVICE *dstnexthop = psPib>u16CoordShortAddr;return TRUE;

\# else //router or coor

\#ifndef I_AM_COORDINATOR

If

(!IsMyDescendant( gsNWK_Para.gsNIB.u16nwkNetwork Address,dstaddr, gsNWK_Para.gNwkDepth ))

\{

$*$ dstnexthop $=$ psPib->u16CoordShortAddr; return TRUE;

\}

\#endif

ChildPtr = gsNWK_Para.neighbortablehead;

while(ChildPtr != NULL)

\{

if $($ ChildPtr->u8Relationship == NEIGHBOR_CHILD $)$ \{

if $\quad(($ ChildPtr->u16Addr $\quad==$

dstaddr)||IsMyDescendant(ChildPtr-

>u16Addr,dstaddr,gsNWK_Para.gNwkDepth+1)))

$\{*$ dstnexthop $=$ ChildPtr- $>$ u 16Addr; return TRUE;

$$
\text { \} }
$$$$
\text { \} }
$$

$>$ next;

ChildPtr $=($ NWK_NeighborTable_s $*)$ ChildPtr-

\}//end while

return FALSE;

\#endif

\}

c) End device: After the initialization, the end devices determine whether to join the network successfully after scanning the beacon frame. If the child nodes cannot successfully join the network, they will rejoin the network; if the child nodes can successfully join the network, they will transmit these corresponding parameters in order to control the LEDs.

\section{SYSTEM TESTING AND RESUlTS}

The intelligent lighting system is tested in the rooms and hallways of the building based on ZigBee tree structure including PAN coordinator, router and end device. The system can perform the smart control according to the light intensity values. Meanwhile, the parameters are displayed on PC such as light intensity and working state of nodes in real time. The test results are shown as in Figure 5.(125cd, $155 \mathrm{~cd}, 180 \mathrm{~cd})$, where LEDs are controlled by PWM according to indoor brightness:

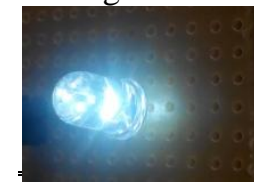

$125 \mathrm{~cd}$

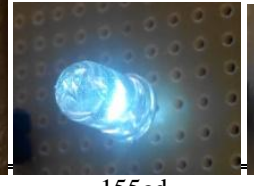

$155 \mathrm{~cd}$

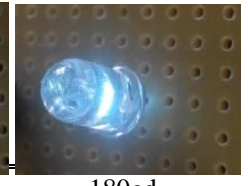

$180 \mathrm{~cd}$
Figure 5. System testing results

\section{CONCLUSION}

The devices in a WPAN must be low cost and operate for a long time from a battery. ZigBee is a technology now being deployed for wireless personal area networks. In this paper, ZigBee technology and the basic tree network topology structure are introduced. On the basis of introduction, the paper discussed a kind of intelligent lighting monitoring system scheme and implement method based CC2530, including hardware and software design. The system can collect the light intensity signals of LEDs in real time; perform PWM and wireless communications between PAN coordinator, router and end devices based on ZigBee tree structure. The article demonstrated the feasibility of the scheme through some corresponding experiments, where the LEDs can be controlled intelligently according to indoor light intensity. Successful implementation of ZigBee based lighting monitoring system provides a solution for convenient lighting work and thus saving energy, as well as cost.

\section{ACKNOWLEDGMENT}

The work was financially supported by the Education Department of Liaoning Province Science and Technology Research Projects (No.L2011123).

\section{REFERENCES}

[1] Alexandru Lavric, Valentin Popa, Codrin Males, Ilie Finis. "A performance study of ZigBee wireless sensors network topologies for street lighting control systems,"2012 International Conference on Selected Topics in Mobile and Wireless Networking. Avignon,: pp. 130-133,2012.

[2] Chi-Hung Hung, Ying-Wen Bai, Ren-Yi Tsai. "Digital Control for Home Lighting System with ZigBee Communication," 2011 IEEE International Conference on Consumer Electronics. Las Vegas, NV,pp. 763-764,2011.

[3] Wei LIU, Yuhua YAN. "Application of ZigBee Wireless Sensor Network in Smart Home System,".International Journal of Advancements in Computing Technology. 3(5),pp. 154-160. 2011

[4] Y.U. Lee, S. Baang, J. Park, Z. Zhou, M. Kavehrad. "Hybrid Positioning with Lighting LEDs and Zigbee Multihop Wireless Network," Proc. SPIE 8282, Broadband Access Communication Technologies VI. San Francisco, California, USA ., 8282,pp. 1-7. 2012

[5] IEEE 802.15.4. Part 15.4, Wireless sedium access control (MAC) and physical layer (PHY) specifications for low-rate wireless personal area networks (LR-WPANs). New York: Institute of Electrical and Electronic Engineers, Inc, 2003.

[6] ZIGBEE ALLIANCE. ZigBee Specification Version 1.0 [EB/OL]. . http://www.zigbee.org.,2004

[7] ZIGBEE ALLIANCE.ZigBee Specification Version1.1[EB/OL]. http://www.zigbee.org.2006

[8] ZIGBEE ALLIANCE. ZigBee-Specification 2007 [EB/OL]. http://www.zigbee.org.2007

[9] ZIGBEE ALLIANCE, ZigBee-specification 2008 [EB/OL]. http://www.zigbee.org.2008

[10] F. Cuomo, E. Cipollone, A. Abbagnale. "Performance analysis of IEEE802.15.4 wireless sensor networks: an insight into the topology formation process". Computer networks. 53(18), pp. 3057 - 3075. 2009, 